\title{
PROOF OF A THEOREM OF HALL
}

\section{HARRY GOHEEN}

In the Journal of the London Mathematical Society for July, 1937, Mr. Philip Hall gave a proof of the theorem, "If a group $G$ of order $g$ has a subgroup of order $m$ for every divisor $m$ of $g$ such that $(m, g / m)=1$, then $G$ is a soluble group." The proof is a very simple one in contrast to the rather difficult proof of the converse theorem which Hall had published in the same journal for April, 1927. It seems worthwhile to give a simpler proof of this converse.

THEOREM. If $g$ is the order of a soluble group $G$ and $m$ is a divisor of $g$ such that $m$ and $g / m$ are relatively prime, then $G$ has a subgroup of order $m$ and furthermore all the subgroups of order $m$ in $G$ are conjugate un$\operatorname{der} G$.

Proof. Since the theorem is true by default for prime power groups, let us suppose that it is true for all soluble groups of orders less than $g$ and use the method of complete induction. Then the theorem is true for an invariant subgroup $G^{\prime}$ of prime index $r$ in $G$.

If $r$ divides $g / m$, then $G^{\prime}$ has a subgroup of order $m$. Since $G^{\prime}$ is invariant and $(m, r)=1$, every element of order dividing $m$ in $G$ must be in $G^{\prime}$. Hence all the subgroups of order $m$ in $G$ must be in $G^{\prime}$ where by hypothesis they form a complete set of conjugates under $G^{\prime}$ and consequently a complete set of conjugates under $G$.

If $r$ divides $m$, then $G^{\prime}$ has a complete set of conjugates of order $m / r$. Since $G^{\prime}$ is invariant, the subgroups of order $m / r$ in $G^{\prime}$ are a complete set of conjugates under $G$. Let $M^{\prime}$ be one of these subgroups of order $m / r$ in $G^{\prime}$.

If $M^{\prime}$ is the only subgroup of order $m / r$ in $G^{\prime}$, then, it must be invariant in $G$. Then the quotient group is of order $\mathrm{rg} / \mathrm{m}$ and since $r$ is a Sylow divisor of the order of this quotient group, it has a subgroup of order $r$. Then $G$ has a subgroup of order $r \cdot m / r$ or $m$. On the other hand if $M^{\prime}$ is not the only subgroup of order $m / r$ in $G^{\prime}$, let it be one of $k$ subgroups of order $m / r$ in $G^{\prime}$. Then $k$ divides the order $g / r$ of $G^{\prime}$ and the normalizer of $M^{\prime}$ in $G$ is of order. $g / k$ divisible by $m$. Since this normalizer is a soluble group of order $g / k$ less than $g$, it has a subgroup of order $m$.

There remains only to show, for the case $r$ divides $m$, that all the subgroups of order $m$ in $G$ are conjugate under $G$. Let $M$ be a subgroup of order $m$. If there is no other subgroup of order $m$ in $G$, then the theorem is true by default. However, if $M_{1}$ is another subgroup 
of order $m$ in $G$ it will be shown that $M$ and $M_{1}$ are conjugate under $G$.

Let the crosscut of $M$ and $G^{\prime}$ be $\Gamma$ of order $\gamma$ and the crosscut of $M_{1}$ and $G^{\prime}$ be $\Gamma_{1}$ of order $\gamma_{1}$. Then since $G$ is generated by $M$ and $G^{\prime}$ as well as by $M_{1}$ and $G^{\prime}$ and since $G^{\prime}$ is invariant under both $M$ and $M_{3}$ we have

$$
(g / r)(m) / \gamma=g, \quad(g / r)(m) / \gamma_{1}=g
$$

whence

$$
\gamma=\gamma_{1}=m / r .
$$

Then since $\Gamma$ and $\Gamma_{1}$ are of order $m / r$, they are conjugate under $G^{\prime}$. If $S^{-1} \Gamma_{1} S=\Gamma$, then $S^{-1} M_{1} S$ and $M$ have a common invariant subgroup $\Gamma$. If $\Gamma$ is invariant in $G$, the quotient group $G / \Gamma$ is of order $g r / m$. Since $r$ is a Sylow divisor, the subgroups $M / \Gamma$ and $S^{-1} M_{1} S / \Gamma$ of order $r$ are conjugate under $G / \Gamma$ and hence $M$ and $S^{-1} M_{1} S$ are conjugate under $G$ as was to be proved. If, however, $\Gamma$ is not invariant under $G$, its normalizer is a proper subgroup of $G$ containing $M$ and $S^{-1} M_{1} S$ which are therefore conjugate under the normalizer of $\Gamma$ as was to be proved.

Reed College 